\title{
Myelodysplastic Syndrome Secondary to Multiple Myeloma: A Case Report and Literature Review
}

\author{
Wanqiu Zhang, Qianshan Tao, Yinwei Li, Yin Pan, Qing Zhang, Fan Wu, Zhimin Zhai* \\ Department of Hematology, Hamatological Research Center, The Second Affiliated Hospital of Anhui Medical University, Hefei, \\ China \\ Email: *zzzm889@163.com
}

How to cite this paper: Zhang, W.Q., Tao, Q.S., Li, Y.W., Pan, Y., Zhang, Q., Wu, F. and Zhai, Z.M. (2021) Myelodysplastic Syndrome Secondary to Multiple Myeloma: A Case Report and Literature Review. Journal of Biosciences and Medicines, 9, $39-43$.

https://doi.org/10.4236/jbm.2021.93005

Received: January 8, 2021

Accepted: March 19, 2021

Published: March 22, 2021

Copyright $\odot 2021$ by author(s) and Scientific Research Publishing Inc. This work is licensed under the Creative Commons Attribution International License (CC BY 4.0).

http://creativecommons.org/licenses/by/4.0/

\begin{abstract}
Background: With the prolongation of survival in recent years, the accumulation of toxic and side effects of therapeutic drugs and the concomitant drug-related adverse reactions were reported in recent years. However, myelodysplastic syndrome secondary to multiple myeloma is rare. Objective: To improve the understanding of myelodysplastic syndrome secondary to multiple myeloma. Methods: The clinical data of a patient with myelodysplastic syndrome secondary to multiple myeloma after treatment were analyzed, and the related literature was reviewed. Results: A 54-year-old male patient was diagnosed as multiple myeloma in February 2014. After three courses of first-line induction chemotherapy, he achieved complete remission and received two courses of consolidation treatment. After that, he continued to take thalidomide orally. The disease recurred 13 months after complete remission (CR) with 6q+ karyotype change in 37 months. 21q- karyotype change was found in 39 months. The patient was finally diagnosed as treatment-related secondary myelodysplastic syndrome. Due to the poor effect of chemotherapy, the disease continued to deteriorate. Conclusion: In the course of multiple drug treatment, clinicians should pay attention to the changes of molecular genetics and the treatment-related secondary tumor.
\end{abstract}

\section{Keywords}

Multiple Myeloma, Myelodysplastic Syndrome, Karyotype, Thalidomide

\section{Introduction}

Multiple myeloma (MM) is a type of plasma cell tumor, which is characterized by the proliferation of clonal plasma cells in bone marrow and the positive de- 
tection of monoclonal protein in blood or urine, leading to the dysfunction of related organs [1]. MM is the second most common malignant tumor, accounting for about $1 \%$ of tumor diseases [2]. At present, the main treatment methods of MM were increasing, including proteasome inhibitors, immunomodulators, alkylating agents and hematopoietic stem cell transplantation. With the prolongation of survival, the accumulation of toxic and side effects of therapeutic drugs and the concomitant drug-related adverse reactions were reported in recent years. However, myelodysplastic syndrome secondary to multiple myeloma is rare. The increased risk of secondary MDS (s-MDS) seriously threatens the long-term prognosis and quality of life of MM patients. Therefore, early detection of patients who are at high risk of secondary tumor will have important guiding significance for the follow-up diagnosis and treatment of patients.

In this study, we report a case of MM with normal karyotype of bone marrow at the initial diagnosis. During the treatment, there were $6 \mathrm{q}+$ and $21 \mathrm{q}-$ chromosome changes. Two months later, s-MDS was diagnosed.

\section{Case Presentation}

A 59-year-old man was admitted to our hospital in February 2014 due to "chest and back pain for 20 days". After hospitalization, blood routine examination show hemoglobin was $62 \mathrm{~g} / \mathrm{L}$. Immunoglobulin shows IgA was $63.51 \mathrm{~g} / \mathrm{L} . \beta 2$ microglobulin was $13.80 \mathrm{mg} / \mathrm{L}$. blood light chain show $\kappa$ chain was $322 \mathrm{mg} / \mathrm{L}$, $\lambda$ chain was $2090 \mathrm{mg} / \mathrm{L}, \kappa / \lambda$ ratio was 0.154 . Blood immunoprotein fixed electrophoresis show IgA- $\lambda$ type $M$ albuminemia. Bone marrow cell morphology shows $54.5 \%$ of immature plasma cells. Bone marrow immunotyping shows $43.8 \%$ abnormal plasma cells with CD38+ CD138+ CD19-CD56+ CD45 dimSSC++ +++. Chromosome karyotype analysis shows 46, XY [20]. The patient was diagnosed with MM (IGA- $\lambda$ type, DS stage-III, ISS stageIII).

After 3 courses of first-line induction chemotherapy, the patient achieved complete response (CR) in July 2014. Then he was consolidated for 2 courses with the original induction regimen. After that, the patients were advised to follow up regularly, but they did not continue the treatment due to economic reasons. He only took thalidomide monotherapy and refused the autologous hematopoietic stem cell transplantation.

13 months after CR, the patient relapsed and then received induction therapy with various regimens, but the curative effect only reached stable disease (SD). At 37 months after CR, the morphology of bone marrow cells showed that bone marrow hyperplasia was still active with $17.5 \%$ immature plasma cells. At the same time, the karyotype of bone marrow chromosome shows $46, \mathrm{XY}$, add (6) (q27) [20]. The result of comprehensive assessment was progressive disease (PD).

39 months after CR, the blood routine showed white blood cell (WBC) count was $1.88 \times 10^{9} / \mathrm{L}$, neutrophil count was $0.43 \times 10^{9} / \mathrm{L}$, hemoglobin was $75 \mathrm{~g} / \mathrm{L}$, 
platelet count was $23 \times 10^{9} / \mathrm{L}$. Bone marrow cell morphology showed active proliferation, 9\% immature plasma cells and 16\% myeloid primordial cells, accompanied with myeloid hematopoiesis. Bone marrow chromosome karyotype showed 46, XY, add (6) (q27), del (21) (q22) [20]. Bone marrow TP53 gene was negative. In summary, s-MDS was diagnosed (RAEB-2, IPSS high risk group, IPSS-R extremely high risk group). Due to the poor physical condition at that time, the patient could not tolerate chemotherapy. The patients were treated with proteasome inhibitor for one course. 42 months after CR, the morphology of bone marrow cells showed 19\% immature plasma cells and 11\% myeloid primordial cells. The karyotype of bone marrow chromosomes showed 46, XY, add (6) (q27), del (21) (q22) [20]. After that, the patients were treated with the chemotherapy regimen consideration to both MM and MDS, but the peripheral blood picture of the patients continued to deteriorate. After chemotherapy, minimal residual disease (MRD) of peripheral blood showed 13\% of myeloid progenitor cells, so the possibility of acute myeloid leukemia (AML) was considered. After that, the patient refused further treatment and discharged automatically. After that, the patient was lost to follow-up.

The course of the disease was 48 months from diagnosis to final deterioration. Abnormal karyotype was found in the 42 months after diagnosis. 16\% of myeloid progenitor cells were found months after diagnosis. From the diagnosis of MM to the diagnosis of secondary MDS, the total dose of thalidomide was gradually increased from $100 \mathrm{mg} / \mathrm{d}$ to $400 \mathrm{mg} / \mathrm{d}$ within the tolerable range of patients.

\section{Discussion}

A large number of literatures have reported that the risk of secondary hematological tumors in tumor patients is significantly higher than that in ordinary people [3] [4] [5]. In recent years, with the prolongation of the survival time in MM patients, the incidence rate of MM related second tumors was increased [6]. Studies show that there is a causal relationship between the treatment of primary malignant tumors and the incidence of secondary tumors. The alkylating agent based therapy combined with immunomodulatory drugs will increase the risk of secondary tumor, among which lenalidomide is the most reported [7] [8]. In the evaluation and analysis of MM patients receiving various chemical regimens, the risk factors of secondary MDS or AML included male patients, old age and using thalidomide or lenalidomide [9].

In this study, the patient was treated with cyclophosphamide, ifosfamide and etoposide, and the patients had been taking thalidomide orally almost all the time during the course of the disease. The dose gradually increased from 100 $\mathrm{mg} / \mathrm{d}$ to $400 \mathrm{mg} / \mathrm{d}$ at the maximum, and there was no obvious adverse reaction. It has been reported that alkylating agent combined with thalidomide may increase the risk of secondary tumor in MM patients. But for this patient, the course of three kinds of chemotherapy drugs was short except thalidomide. 
Therefore, thalidomide can affect the bone marrow microenvironment through a variety of cell regulatory factors, and improve the tumor killing effect of $\mathrm{T}$ cells and NK cells. However, whether it is accompanied by the activation of proto oncogenes or pathological changes of bone marrow hematopoietic microenvironment, and then induce the occurrence of the second tumor needs further study.

In this study, the patient was diagnosed with MDS two months after karyotype changed. A study [10] analyzed 41 cases of myeloid tumor secondary to plasma cell tumor and the result shows that the median time is 60 months from MM treatment to diagnosis of Myeloid Neoplasms. Most patients had abnormal changes of chromosome karyotype, especially $-5(\mathrm{q}) /-7(\mathrm{q}) .-5(\mathrm{q}) /-7(\mathrm{q})$ is directly related to a variety of chemotherapy regimens, especially with the use of alkylating agents such as melphalan or cyclophosphamide. In this study, $6 \mathrm{q}+$ and 21q- karyotypes were found. Chromosome 6 abnormality is relatively rare in the genetic changes of hematological malignancies. It has been reported that it can be seen in B-cell tumors, such as acute lymphoblastic leukemia (ALL), which has monitoring significance for the prognosis of the disease [11]. Chromosome 6 is the most frequent site of loss of heterozygosity in tumors, and several frequently lost hot spots 6p21, 6q21, 6q24-q25 and 6q27 are involved in the occurrence and development of tumors [12]. This is not consistent with $6 \mathrm{q}+$ in this case, so the correlation between $6 \mathrm{q}+$ and secondary MDS still need more clinical studies. In addition, the patient's chromosome $21 \mathrm{q}-$ was associated with Down's syndrome, some complex diseases (familial compound hyperlipidemia, bipolar affective disorder), some single gene genetic disorders (amyotrophic lateral sclerosis, autoimmune polyadenopathy, Alzheimer's disease, etc.) [13], solid tumors (cervical cancer, lung cancer, breast cancer, etc.) and leukemia. Acute myeloid leukemia 1 (AML1) is located on the long arm of chromosome 21. AML1 protein is also known as runt related transcription factor 1 (RENX1), which plays an important role in the growth and angiogenesis of hematopoietic cells. Deletion of RUNX1 gene located in 21q22 region can lead to myelodysplastic syndrome.

In conclusion, patients with multiple myeloma should be monitored blood routine test and chromosome karyotype during the treatment. Patients with unexplained abnormal blood and abnormal chromosome karyotype should be alert to the occurrence of secondary hematological second tumor.

\section{Consent}

Written informed consent was obtained from the patient for publication of this article and any accompanying images. A copy of the written consent is available for review by the Editor of this journal.

\section{Acknowledgements}

This work is supported by the National Natural Science Foundation of China (grant No. 81670179). The authors thank the patient and his families and all the 
investigators, including the physicians, nurses, and laboratory technicians in this study.

\section{Conflicts of Interest}

The authors declare that they have no competing interests.

\section{References}

[1] Palumbo, A. and Anderson, K. (2011) Multiple Myeloma. The New England Journal of Medicine, 364, 1046-1060. https://doi.org/10.1056/NEJMra1011442

[2] Raab, M.S., Podar, K., Breitkreutz, I., et al. (2009) Multiple Myeloma. The Lancet, 374, 324-339. https://doi.org/10.1016/S0140-6736(09)60221-X

[3] Li, J.X., Liu, J.R., Chen, M.L., et al. (2016) Three Patients with Multiple Myeloma Developing Secondary Lymphoblastic Leukemia: Case Reports and Review of the Literature. Tumori Journal, 102, S131-S136. https://doi.org/10.5301/tj.5000377

[4] Piszcz, J., Bolkun, L., Cichocka, E., et al. (2012) Secondary Acute Lymphoblastic Leukaemia in a Multiple Myeloma Patient. Contemporary Oncology, 16, 593-595. https://doi.org/10.5114/wo.2012.32497

[5] Gonzalez, M.M., Kidd, L., Quesada, J., et al. (2013) Acute Myelofibrosis and Acute Lymphoblastic Leukemia in an Elderly Patient with Previously Treated Multiple Myeloma. Ann Clin Lab Sci, 43, 176-180.

[6] Wang, Y., Yang, F., Shen, Y., et al. (2015) Maintenance Therapy with Immunomodulatory Drugs in Multiple Myeloma: A Meta-Analysis and Systematic Review. Journal of the National Cancer Institute, 108, Article ID: djv342. https://doi.org/10.1093/jnci/djv342

[7] McCarthy, P.L., Holstein, S.A., Petrucci, M.T., et al. (2017) Lenalidomide Maintenance after Autologous Stem-Cell Transplantation in Newly Diagnosed Multiple Myeloma: A Meta-Analysis. Journal of Clinical Oncology, 35, 3279-3289. https://doi.org/10.1200/JCO.2017.72.6679

[8] McCarthy, P.L., Owzar, K., Hofmeister, C.C., et al. (2012) Lenalidomide after Stem-Cell Transplantation for Multiple Myeloma. The New England Journal of Medicine, 366, 1770-1781. https://doi.org/10.1056/NEJMoa1114083

[9] Usmani, S.Z., Sawyer, J., Rosenthal, A., et al. (2013) Risk Factors for MDS and Acute Leukemia Following Total Therapy 2 and 3 for Multiple Myeloma. Blood, 121, 4753-4757. https://doi.org/10.1182/blood-2012-11-466961

[10] Reddi, D.M., Lu, C.M., Fedoriw, G., et al. (2012) Myeloid Neoplasms Secondary to Plasma Cell Myeloma: An Intrinsic Predisposition or Therapy-Related Phenomenon? A Clinicopathologic Study of 41 Cases and Correlation of Cytogenetic Features with Treatment Regimens. American Journal of Clinical Pathology, 138, 855-866. https://doi.org/10.1309/AJCPOP7APGDT9JIU

[11] Lawce, H. and Olson, S. (2009) FISH Testing for Deletions of Chromosome 6q21 and 6q23 in Hematologic Neoplastic Disorders. J Assoc Genet Technol, 35, 167-169.

[12] Katagiri, T., Sato-Otsubo, A., Kashiwase, K., et al. (2011) Frequent Loss of HLA Alleles Associated with Copy Number-Neutral 6pLOH in Acquired Aplastic Anemia. Blood, 118, 6601-6609. https://doi.org/10.1182/blood-2011-07-365189

[13] Melis, D., Genesio, R., Cappuccio, G., et al. (2011) Mental Retardation, Congenital Heart Malformation, and Myelodysplasia in a Patient with a Complex Chromosomal Rearrangement Involving the Critical Region 21q22. American Journal of Medical Genetics A, 155, 1697-1705. https://doi.org/10.1002/ajmg.a.33976 